\title{
Scopolamine-induced Migraine Like Headache
}

\author{
Ken Ikeda, Yuji Kawase, Takanori Takazawa, Konosuke Iwamoto, Hirono Ito, \\ Kiyoko Murata, Ryuta Sato, Tetsuro Nagaoka, Tokinori Sekine, Riya Nagata, \\ Yoshikazu Nakamura, Takehisa Hirayama, Yuichi Ishikawa, Ken Miura, \\ Yasuhiro Yoshii and Yasuo Iwasaki
}

\begin{abstract}
Objective Scopolamine butylbromide (SB), a muscarinic receptor antagonist, is used commonly in gastric X-ray examination in the physical check-up in Japan. This study describes clinical features of SB-induced headache.

Patients and Methods SB-induced headache was defined as headache that started within 20 minutes after intramuscular administration of SB (20 mg/body). The Primary and the secondary headaches were diagnosed according to the ICHD-II criteria. SB-induced headache was classified as headache induced by acute substance use or that due to exposure (ICHD-II code 8.1). Clinical features and background of subjects with SBinduced headache were analyzed. We also estimated the frequency of SB-related headache between migraineurs and non-migraineurs.

Results A total of 54 subjects (39 women and 15 men) experienced SB-induced headache. All subjects had the present history of migraine. Nine subjects had $\geq 2$ times of the headache. Mean age (SD) was 46.2 (9.7) years [46.2 (9.7) for women and 46.3 (10.0) for men]. Clinical hallmarks of headache showed that pulsating / throbbing pain occurred in diffuse or bilateral head sites. Headache worsened at 20-30 minutes from the onset and persisted for 6-18 hours, and ameliorated gradually 8 hours later. All subjects had repeated nausea and vomiting. Severity of headache revealed severe degree requiring complete bed rest in 50 subjects (92.6\%). SB-induced headache had similar characteristics as migraine without aura (MO) attacks. Liver and renal functions were normal in all SB-related migraineurs. They had no allergic history of medication and food. In 1,865 non-migraine controls, one healthy subject had a mild degree of migraine like headache triggered by SB injection.

Conclusion SB triggers a severe degree MO like headache or worsens pre-existing migraine in some migraineurs. SB-induced headache could contribute to disequilibrium between acetylcholine and other neuropeptides. We should use SB more carefully as it can be an aggravating drug of migraine.
\end{abstract}

Key words: scopolamine butylbromide, secondary headache, migraine, anti-cholinergic drug, muscarinic receptor

(Inter Med 48: 681-685, 2009)

(DOI: 10.2169/internalmedicine.48.1941)

\section{Introduction}

Migraine is a common disorder particularly in subjects of working age. A migraine attack is triggered by several components, such as menstruation, irregular sleep, weather, foods and acute substance use. Pharmacological studies point out that dopamine is associated with migraine and dopaminergic stimulation induces migraine attacks in some migraineurs (1-3). Clinical trails of donepezil, a cholinesterase inhibitor for a therapeutic drug in Alzheimer's disease, show successful prophylaxis of migraine $(4,5)$.

Scopolamine butylbromide (SB) is an anti-muscarinic drug. This drug is widely used as pre-medication on gastric 
X-ray examination in Japanese physical check-up. We encountered migraine sufferers who had migraine like headache after SB administration. The present study of case series aimed to exhibit clinical features and background of subjects with SB-induced headache.

\section{Patients and Methods}

\section{Diagnosis and classification of SB-induced head- ache}

SB was injected into healthy adults ( $<70$ years of age) as a part of the typical procedure for gastro-duodenal X-ray examination in PL Tokyo Health Care Center, Tokyo, Japan. A board-certified neurologist and headache specialist (K.I.) performed an interview of headache history and neurological examination when subjects complained of acute headache after SB injection. SB-induced headache was defined as headache started within 20 minutes after intramuscular administration of SB (20 mg/body). Primary and the secondary headaches were diagnosed according to the ICHD-II criteria (6). SB-induced headache was classified into headache induced by acute substance use or exposure (ICHD-II code 8.1).

\section{Clinical background of SB-induced headache}

Clinical background and profile of SB-induced headache were assessed for age, sex, headache history, pain type, headache site, severity and persistent time. Duration of this study was between July 2005 and November 2006. During this period, we investigated whether headache occurred in age-matched non-migraine controls. Consecutive 1,865 controls (615 women and 1250 men) were diagnosed as nonmigraineurs examined by a board-certified neurologist and headache specialist (K.I.).

\section{Results}

\section{Clinical profile of SB-induced headache}

A total of 54 subjects (39 women and 15 men) experienced SB-induced headache. Nine subjects experienced $\geq 2$ times of headache induced by SB injection on their medical records of physical check-up. All subjects suffered from migraine. Clinical characteristics of SB-induced headache were shown in Table 1. Pulsating/throbbing headache happened in diffuse or bilateral portion of the head and the headache become worse at 20-30 minutes from the onset. Nausea and vomiting accompanied in all subjects. All subjects had no migraine aura-like symptoms. The severity of headache classified as follows: 50 subjects $(92.6 \%)$ had severe degree requiring complete bed rest and cessation of physical checkup under or after gastric X-ray, 5 subjects (7.4\%) exhibited moderate degree that they were able to walk at 3-4 hours after medication of a pain killer. Those clinical aspects revealed that SB-induced headache had similar characteristics
Table 1. Clinical Features of SB-induced Headache

\begin{tabular}{ll}
\hline Subjects with SB-induced headache & \\
\hline Total number & 54 migraineurs \\
Sex, n (\%) & $39(72.2)$ \\
Women & $15(27.8)$ \\
Men & $54(100)$ \\
Characteristics of headache, $\mathrm{n}(\%)$ & $54(100)$ \\
Pulsating / throbbing & none $(0)$ \\
Nausea / vomiting & $54(100)$ \\
Aura symptoms & \\
MO like headache & $54(100)$ \\
Bilateral / diffuse & none $(0)$ \\
Unilateral / local & \\
Headache site, $(\%)$ & $50(92.6)$ \\
Severity of attacks, $\mathrm{n}(\%)$ & $4(7.4)$ \\
Moderate degree & none $(0)$ \\
Mild degree & $20-30$ minutes \\
Worsening time from the onset & $6-18$ hours / attack \\
Persistent time
\end{tabular}

MO: migraine without aura

to migraine without aura (MO) in all subjects. Different profile of SB-induced headache and spontaneous migraine revealed that none had migraine with aura (MA) like headache. SB-induced headache always occurred in diffuse or bilateral sites. Pulsating/throbbing headache persisted for at least 6 hours. After 8 hours from the onset, headache gradually reduced in all subjects. All subjects had no recurrence of headache the following day. Thus, SB-induced headache was diagnosed definitely as headache as an acute adverse event attributed to medication used for other indications (ICHD-II code 8.1.10).

One control subject $(0.05 \%)$ complained of migraine like headache among 1,865 non-migraineurs. The severity of headache was a mild degree without vomiting that spontaneously recovered at 2 hours after SB injection.

\section{Clinical background of migraineurs with SB-induced headache}

Clinical background of 54 migraine sufferers with SBinduced headache was shown in Table 2. Usual migraine subtype revealed $48 \mathrm{MO}$ sufferers (34 women and $14 \mathrm{men}$ ) and 6 MA sufferers (5 women and 1 man). Mean age (SD) was 46.2 (9.7) years [46.2 (9.7) in women and 46.3 (10.0) in men]. Mean onset age of migraine (SD) was 22.6 (9.3) years [22.3 (8.7) in women and 22.5 (10.9) in men]. Mean duration of migraine (SD) was 23.7 (11.3) years [23.6 (12.5) in women and 23.9 (7.9) in men]. Family history of migraine disclosed in 28 subjects $(51.9 \%)$. Frequency of migraine attack was $\geq 1$ attack per month in 29 subjects (20 women and 9 men) and $<1$ attack per month in 25 (19 women and $6 \mathrm{men})$. As for migraine severity, 19 subjects had severe degree of migraine defined as requiring bed rest or absence from work. Moderate degree (50\% reduction of job ability) and mild degree were 21 subjects and 14 subjects, respectively. Trigger factors of migraine during daily 
Table 2. Clinical Background of Migraine Sufferers with SB-induced Headache

\begin{tabular}{llll}
\hline & Total & Women & Men \\
\hline Number & 54 subjects & 39 subjects & 15 subjects \\
Age, mean years (SD) & $46.2(9.7)$ & $46.2(9.7)$ & $46.3(10.0)$ \\
Onset age of migraine, mean years (SD) & $22.6(9.3)$ & $22.3(8.7)$ & $22.5(10.9)$ \\
Duration of migraine, mean years (SD) & $23.7(11.3)$ & $23.6(12.5)$ & $23.9(7.9)$ \\
Subtype of migraine, n (\%) & & & $1(6.7)$ \\
$\quad$ MA sufferers & $6(11.1)$ & $5(12.8)$ & $14(93.3)$ \\
$\quad$ MO sufferers & $48(88.9)$ & $34(87.2)$ & $9(60.0)$ \\
$\quad$ & & $6(40.0)$ \\
$\quad$ Frequency of migraine attacks, n (\%) & $29(53.7)$ & $20(51.3)$ & $19(48.7)$ \\
$\quad$ 1 attack / month & $25(46.3)$ & & $3(20.0)$ \\
$\quad$ Severe degree & & $16(41.0)$ & $10(66.7)$ \\
$\quad$ Moderate degree & $19(35.2)$ & $11(33.3)$ & $2(13.3)$ \\
$\quad$ Mild degree & $21(38.9)$ & $12(30.8)$ & $6(40.0)$ \\
\hline
\end{tabular}

MA: migraine with aura; MO: migraine without aura

life were irregular sleep (55.6\%), crowdedness (44.4\%), weather changes $(28.9 \%)$, menstruation $(33.3 \%)$, stress/tension $(33.3 \%)$, alcohols $(17.9 \%)$ and lack of foods $(8.9 \%)$. There was no allergic history of drugs and foods in migraineurs with SB-induced headache. Physical check-up suggested normal liver and renal function in those subjects. Those results showed that the SB-induced MO like headache was not caused by hypersensitivity, abnormal metabolism or intoxication of SB injection.

\section{Case report}

Typical cases with SB-induced headache are described here.

Case 1: A 45-year-old woman developed migraine at 25 years of age. The subtype of migraine was MO and frequency of migraine attacks was six times/year. There was no allergic history of medication and food. When gastric X-ray examination was performed at 10 minutes after intramuscular administration of SB, she noticed a mild degree of pulsating pain in the bilateral head site. Twenty minutes later, her headache became worse with nausea and vomiting. She took bed rest and her headache improved gradually after 6 hours and resolved by the following morning.

Case 2: A 46-year-old woman suffered from migraine at 20 years of age. Subtype of migraine was MA and frequency of migraine attacks was one or two times/month. There was no allergic history of medication or food. At 10 minutes after intramuscular administration of SB, she noticed mild degree of pulsating and diffuse headache. Usual visual aura symptoms were not presented. At 30 minutes after SB injection, she needed bed rest for severe headache and vomiting. The severe headache persisted for 8 hours. She recovered from headache completely by the following morning.

\section{Discussion}

The present study showed moderate or severe MO like headaches after acute exposure of SB in migraineurs. As for the differing profile between spontaneous migraine and SBinduced headache, SB-associated migraine like headache exhibited no visual aura symptoms. Throbbing pain started in diffuse or bilateral head sites. The clinical course of headache disclosed that SB-induced headache was classified as definite headache as an acute adverse event attributed to medication used for other indications (ICHD-II code 8.1.10). Otherwise, only one non-migraineur experienced a mild degree of migraine like headache after SB administration. The correct incidence of migraine like attacks after SB injection remains unclear in the present study. Our presumed incidence of SB-induced headache was estimated as 47 subjects (8.9\%) among 529 consecutive migraineurs, as compared to $0.05 \%$ of non-migraine controls. Those data point out that SB administration induces MO like headache or worsens pre-existing migraine in migraineurs, in comparison with non-migraineurs.

SB possesses anti-cholinergic effects mediated by muscarinic receptors. This drug is applied as a transdermal patch for the prevention of seasickness in Israel. Previous case report shows that a 20-year-old healthy man developed recurrent migraine after transdermal SB intoxication (7). In the present study of SB-induced headache, 54 migraineurs and one non-migraineur had no clinical course and past history of SB intoxication. Those subjects received SB at fasting state of one overnight. The fasting condition may induce or exaggerate headache attributed to SB injection. After intramuscular SB administration, anti-muscarinic effects start at 8-10 minutes and the half-life time is approximately 8 hours. The onset and persistence of SB-induced headache is similar to pharmacodynamics of SB.

Acetylcholine acts on dural vessels, leading to vascular dilatation. This fact proposes one possibility whether migraine like headache is associated with cholinergic vascular dilatation. In an experimental study of rat dural vasodilation, intravenous administration of scopolamine $(2 \mathrm{mg} / \mathrm{kg})$ markedly inhibited dilation of the middle meningeal artery 
branch after acetylcholine injection (8). Mecamylamine, a nicotinic receptor antagonist, does not inhibit acetylcholineinduced dilation of the middle meningeal artery branch. Those results disclose that cholinergic dilation of dural arteries is mediated by muscarinic receptors, but not nicotinic receptors (8). In another experiment model, scopolamine administration does not respond to dural vasodilation by electric stimulation on the surface of the cranial window. Scopolamine, a muscarinic antagonist, does not influence neurogenic dural vessel dilation in rat electric stimulation model (8). Thus, a different mechanism exists between pharmacological and neurogenic dilation of dural vessels in anesthetized rats. A different previous study also revealed that scopolamine administration does not alter the response of cerebral blood flow to somatosensory stimulation (9). Thus, these experimental studies suggest that SB-induced migraine like headache cannot be precisely explained by meningovascular changes due to direct anti-muscarinic effects of SB.

Recent pathophysiologic mechanism of migraine focuses on the serotonergic role of 5-hydroxytryptamine (5-HT). Several studies suggest the relationship between cholinergic and serotonergic systems in the brain (10-14). Intrathecal and systemic administration of cholinesterase inhibitors produce antinociception in several kinds of animal models (1014). Treatment with physostigmine, a cholinesterase inhibitor, elevates the nociceptive pain threshold in humans (15). Two recent clinical studies of migraine prevention describe that donepezil, an anti-cholinesterase drug, has benefits for migraine prophylaxis $(4,5)$. In the Italian group, the first clinical trail of donepezil was performed in migraineurs. This drug has better preventive effects of migraine, in comparison with propranolol treatment (4). This report of the Italian group also studies anti-nociceptive activities of done- pezil in mouse hot plate test. Of interest, this drug has dosedependent anti-nociceptive effects and the effects are blocked by simultaneous administration of scopolamine on this animal model (4). Another clinical trial of randomized, controlled, double-blind study of donepezil shows that this drug $(10 \mathrm{mg} /$ day) prevents migraine attacks as effective as propranolol LA (160 mg/day) (5). Both clinical trials strongly indicate that cholinergic pathway could contribute to presence and prophylaxis of migraine attacks. Sumatriptan, a $5-\mathrm{HT}_{1 \mathrm{~B} / \mathrm{D}}$ receptor agonist, is used widely as an antimigraine drug. The mechanism of this drug is known to provide antinociceptive effects via the central cholinergic system in several animal species (16). In mouse hot plate and abdominal constriction tests, antinociception of sumatriptan is blocked by atropine, a muscarinic receptor antagonist (17). Those experimental studies support strongly that migraine is associated with the cholinergic neuron network, in addition to the serotonergic pathway in the central nervous system. Thus, the harmful interaction between cholinergic and serotonergic neurons could play a role in the pathogenesis of migraine like headache trigged by SB. Intramuscular administration of SB may induce pharmacological changes of acetylcholine, serotonin and other neuropeptides.

\section{Conclusion}

The present case-series study indicates that acute exposure of SB induces severe MO like headache or worsens preexisting migraine in migraineurs. Although SB is not included in the drug list of ICHD-II that may induce headache or worsen pre-existing headache, we should more carefully consider the use of this drug in migraine sufferers.

\section{References}

1. Peroutka SJ. Dopamine and migraine. Neurology 49: 650-656, 1997.

2. Blin O, Azulay J, Masson G, Aubrespey G, Serratrice G. Apomorphine-induced yawing and migraine patients: Enhanced responsiveness. Clin Neuropharmacol 14: 91-95, 1991.

3. Del Bene E, Poggioni M, DeTommasi F. Video assessment of yawing induced by sublingual apomorphine in migraine. Headache 34: 536-538, 1994.

4. Nicolodi M, Galeotti N, Ghelardini C, Bartolini A, Sicuteri F. Central cholinergic challenging of migraine by testing secondgeneration anticholinesterase drugs. Headache 42: 596-602, 2002.

5. Klapper J, Mathew NT, Abu-Shakra S. Donepezil vs. propranolol for migraine prevention: Results of a randomized, controlled, double-blind, double-dummy clinical trial. Neurology 64 (Suppl. 1): A335, 2005.

6. Headache Classification Subcommittee of the International Headache Society. The International Classification of Headache Disorders. 2nd edition. Cephalalgia 24 (Suppl. 1): 1-160, 2004.

7. Gordon CR, Mankuta D, Shupak A, Spitzer O, Doweck I. Recurrent classic migraine attacks following transdermal scopolamine intoxication. Headache 31: 172-174, 1991.

8. Kowacs F, Williamson DJ, Goadsby PJ. Neurogenic vasodilation of dural blood vessels is not mediated by cholinergic transmission in the anaesthetized rat. Eur J Pharmacol 493: 133-137, 2004.

9. Nakao Y, Gotoh J, Kuang TY, Cohen DM, Pettigrew KD, Sokoloff L. Cerebral blood flow responses to somatosensory stimulation are unaffected by scopolamine in unanesthetized rat. J Pharmacol Exp Ther 290: 929-934, 1999.

10. Bianchi C, Siniscalchi A, Beani L. 5-HT $\mathrm{HA}_{1 \mathrm{~A}}$ agonists increase and $5-\mathrm{HT}_{3}$ agonists decrease acetylcholine efflux from the cerebral cortex of freely moving guinea-pigs. Br J Pharmacol 101: 448452, 1990.

11. Yaksh TL, Driksen R, Harty GJ. Antinociceptive effects of intrathecally injected cholinomimetic drugs in the rat and cat. Eur $\mathrm{J}$ Pharmacol 117: 81-88, 1985.

12. Smith MD, Yang X, Nha JY, Boccafusco JI. Antinociceptive effect of spinal cholinergic stimulation: Interaction with substance $P$. Life Sci 45: 1255-1261, 1989.

13. Naguib M, Yaksh LT. Antinociceptive effects of spinal cholinesterase inhibition and isobolographic analysis of the interaction with $\mu$ and $\alpha_{2}$ receptor systems. Anesthesiology 80: 13381348, 1994.

14. Ghelardini C, Galeotti N, Bartolini A. Loss of muscarinic antinociception by antisense inhibition of $\mathrm{M}_{1}$ receptors. Br J Pharmacol 129: 1633-1640, 2000.

15. Pellandra CL. La geneserine-morphine adjuvant de I'anesthesia 
Inter Med 48: 681-685, 2009 DOI: 10.2169/internalmedicine.48.1941

generale. Lyon Med 151: 653, 1933.

16. Ghelardini C, Galeotti N, Figini M, et al. The central cholinergic system has a role in the antinociception induced in rodents and guinea pigs by the antimigraine drug sumatriptan. J Pharmacol Ex Ther 279: 884-890, 1996.

17. Ghelardini C, Galeotti N, Nicolodi M, Donaldson S, Sicuteri F,
Bartolini A. Involvement of central cholinergic system in antinociception induced by sumatriptan in mouse. Int J Clin Pharmacol Res 17: 105-109, 1997.

(C) 2009 The Japanese Society of Internal Medicine http://www.naika.or.jp/imindex.html 